\title{
Fixed Point Theorems in Complete Cone Metric Spaces over Banach Algebras
}

\author{
Seong-Hoon Cho \\ Department of Mathematics, Hanseo University, 46 hanseo 1ro, Chungnam, 31962, Republic of Korea \\ Correspondence should be addressed to Seong-Hoon Cho; shcho@hanseo.ac.kr
}

Received 4 June 2018; Accepted 26 July 2018; Published 5 August 2018

Academic Editor: Richard I. Avery

Copyright (C) 2018 Seong-Hoon Cho. This is an open access article distributed under the Creative Commons Attribution License, which permits unrestricted use, distribution, and reproduction in any medium, provided the original work is properly cited.

\begin{abstract}
The notion of C-class functions in Banach algebras is introduced. By using such concept, a new fixed point theorem is established. An example to illustrate main theorem is given. Finally, applications of our main result to cyclic mappings and weak contraction type mappings are given.
\end{abstract}

\section{Introduction and Preliminaries}

Huang and Zhang [1] introduced cone metric spaces which are generalizations of metric spaces, and they extended Banach's contraction principle to such spaces, whereafter many authors (for example, [2-6] and references therein) studied fixed point theorems in cone metric spaces.

Recently, Liu and $\mathrm{Xu}$ [7] introduced the notion of cone metric spaces over Banach algebras, which is a modification of the concept of cone metric spaces over real Banach spaces [1], and proved the existence of fixed points for mappings defined on such spaces, and they gave an example that fixed point results in metric spaces and in cone metric spaces are not equivalent.

Very recently, Chandok et al. [8] introduced the concept of TAC-contractive mappings by using the notions of C-class functions and cyclic $(\alpha, \beta)$-admissible mappings and established corresponding fixed point theorems in metric spaces.

In the paper, we introduce the notions of $\mathrm{C}$-class functions and cyclic $(\alpha, \beta)$-admissible mappings in Banach algebras. By using such concepts, we introduce a new contraction. We obtain a new fixed point theorem and give an example to illustrate main result. Finally, we give applications of our main result to cyclic mappings and weak contraction type mappings in cone metric spaces over Banach algebras.

A Banach space $\mathscr{A}$ is called a (real) Banach algebra (with unit) if there exists a multiplication $\mathscr{A} \times \mathscr{A} \longrightarrow \mathscr{A}$ that has the followings properties: for all $x, y, z \in \mathscr{A}, \alpha \in \mathbb{R}$,

(1) $(x y) z=x(y z)$;

(2) $x(y+z)=x y+x z$ and $(x+y) z=x z+y z$;

(3) $\alpha(x y)=(\alpha x) y=x(\alpha y)$;

(4) there exists $e \in \mathscr{A}$ such that $x e=e x=x$;

(5) $\|e\|=1$;

(6) $\|x y\| \leq\|x\|\|y\|$.

An element $x \in \mathscr{A}$ is called invertible if there exists $x^{-1} \in$ $\mathscr{A}$ such that $x x^{-1}=x^{-1} x=e$.

Proposition 1 (see [9]). Let $\mathscr{A}$ be a Banach algebra, and let $x \in \mathscr{A}$. If the spectral radius $\rho(x)$ of $x$ is less than 1, i.e.,

$$
\rho(x)=\lim _{n \longrightarrow \infty}\left\|x^{n}\right\|^{1 / n}=\inf _{n \geq 1}\left\|x^{n}\right\|^{1 / n}<1,
$$

then $e-x \in G(\mathscr{A})$, where $G(\mathscr{A})$ is the set of all invertible elements of $\mathscr{A}$ and

$$
(e-x)^{-1}=\sum_{n=0}^{\infty} x^{n} .
$$

Remark 2. Let $\mathscr{A}$ be a Banach algebra.

Then the following are satisfied: 
(1) for all $x \in \mathscr{A}, \rho(x) \leq\|x\|$;

(2) if the condition $\rho(x)<1$ is replaced by $\|x\|<1$ in Proposition 1, then the conclusion holds.

Consistent with Liu and $\mathrm{Xu}$ [7], the following definitions will be needed in the sequel.

Let $\mathscr{A}$ be a Banach algebra. A subset $P$ of $\mathscr{A}$ is called cone if the following conditions are satisfied:

(1) $P$ is a nonempty and closed subset of $\mathscr{A}$ and $\{0, e\} \subset P$;

(2) $a x+b y \in P$, whenever $x, y \in P$ and $a, b \in \mathbb{R}(a, b \geq 0)$;

(3) $P^{2}=P P \subset P$;

(4) $P \cap(-P)=\{0\}$.

Remark 3. Let $\mathscr{A}$ be a Banach algebra, and $P \subset \mathscr{A}$ be a cone.

If $x \in P$ and $\rho(x)<1$, then $e-x \in G(P)$.

Given a cone $P \subset \mathscr{A}$, we define a partial ordering $\leq$ with respect to $P$ by $x \leq y$ if and only if $y-x \in P$. We write $x<y$ to indicate that $x \leq y$ but $x \neq y$.

For $x, y \in P, x \ll y$ stand for $y-x \in \operatorname{int}(P)$, where int $(P)$ is the interior of $P$. A cone $P$ is called normal if there exists a number $K \geq 1$ such that, for all $x, y \in \mathscr{A},\|x\| \leq K\|y\|$ whenever $0 \leq x \leq y$.

A cone $P$ is called regular if every increasing sequence which is bounded from above is convergent. That is, if $\left\{u_{n}\right\}$ is a sequence such that for some $z \in \mathscr{A}$

$$
u_{1} \leq u_{2} \leq \cdots \leq z
$$

then there exists $u \in \mathscr{A}$ such that

$$
\lim _{n \rightarrow \infty}\left\|u_{n}-u\right\|=0 \text {. }
$$

Equivalently, a cone $P$ is regular if and only if every decreasing sequence which is bounded from below is convergent.

It is well known that every regular cone is normal.

From now on, we assume that $\mathscr{A}$ is a Banach algebra, $P \subset$ $\mathscr{A}$ is a cone with $\operatorname{int}(P) \neq \emptyset$, and $\leq$ is partial ordering with respect to $P$.

Let $X$ be a nonempty set, and let $d: X \times X \longrightarrow \mathscr{A}$ be a map such that

(1) $0 \leq d(x, y)$ for all $x, y \in X$ and $d(x, y)=0$ if and only if $x=y$;

(2) $d(x, y)=d(y, x)$ for all $x, y \in X$;

(3) $d(x, y) \leq d(x, z)+d(z, y)$ for all $x, y, z \in X$.

Then $d$ is called a cone metric on $X$ (with a Banach algebra $\mathscr{A})$ and $(X, d)$ is called cone metric space (with a Banach algebra $\mathscr{A})$.

Remark 4. If $\mathscr{A}=\mathbb{C}$ and $P=\{z \in \mathbb{C}: \operatorname{Re}(z) \geq 0$ and $\operatorname{Im}(z) \geq 0\}$, where $\mathbb{C}$ is the set of all complex numbers, then we have that

(1) $z_{1} \leq z_{2} \Longleftrightarrow\left[\operatorname{Re}\left(z_{1}\right) \leq \operatorname{Re}\left(z_{2}\right)\right.$ and $\operatorname{Im}\left(z_{1}\right) \leq$ $\left.\operatorname{Im}\left(z_{2}\right)\right] \Longleftrightarrow z_{2}-z_{1} \in P$
(2) the complex valued metric [10] is a cone metric.

A sequence $\left\{x_{n}\right\}$ of points in a cone metric space $(X, d)$ converges to a point $x \in X$ (denoted by $\lim _{n \rightarrow \infty} x_{n}=x$ or $\left.x_{n} \longrightarrow x\right)$ if for any $c \in \mathscr{A}$ with $0 \ll c$, there exists $N$ such that for all $n>N, d\left(x_{n}, x\right) \ll c$. A sequence $\left\{x_{n}\right\}$ of points in a cone metric space $(X, d)$ is Cauchy if for any $c \in \mathscr{A}$ with $0 \ll c$, there exists $N$ such that for all $n, m>N, d\left(x_{n}, x_{m}\right) \ll c$. A cone metric space $(X, d)$ is called complete if every Cauchy sequence is convergent.

Remark 5. Let $(X, d)$ be a cone metric space and $\left\{x_{n}\right\} \subset X$ be a sequence and $x \in X$. Then the following are satisfied:

(1) $\lim _{n \rightarrow \infty} x_{n}=x$ if and only if for any $c \in \operatorname{int}(P)$, there exists $N$ such that for all $n>N, c-d\left(x_{n}, x\right) \in \operatorname{int}(P)$;

(2) $\left\{x_{n}\right\}$ is a Cauchy sequence if and only if for any $c \in$ $\operatorname{int}(P)$, there exists $N$ such that for all $n, m>N, c-$ $d\left(x_{n}, x_{m}\right) \in \operatorname{int}(P)$.

Note that if $\lim _{n \rightarrow \infty} d\left(x_{n}, x\right)=0$, then $\lim _{n \rightarrow \infty} x_{n}=x$. The converse is true if $P$ is a normal cone. Also, if $P$ is a normal cone, then $\left\{x_{n}\right\}$ is a Cauchy sequence in $X$ if and only if $\lim _{n, m \rightarrow \infty} d\left(x_{n}, x_{m}\right)=0$.

Let $(X, d)$ be a cone metric space.

Let $\Phi$ be the class of all continuous function $\phi: \operatorname{int}(P) \cup$ $\{0\} \longrightarrow \operatorname{int}(P) \cup\{0\}$ satisfying the following:

$$
\begin{aligned}
& (\phi 1) \phi^{-1}(\{0\})=0 ; \\
& (\phi 2) \phi(t) \ll t \text { for all } t \in \operatorname{int}(P) ;
\end{aligned}
$$

$(\phi 3)$ either $\phi(t) \leq d(x, y)$ or $d(x, y) \ll \phi(t)$ for all $t \in \operatorname{int}(P)$ and $x, y \in X$.

Lemma 6 (see [11]). Let $(X, d)$ be a cone metric space with regular cone $P$ such that $d(x, y) \in \operatorname{int}(P)$ for $x \neq y$. Let $\left\{x_{n}\right\} \subset$ $X$ be a sequence, and let $\phi \in \Phi$.

If the sequence $\left\{x_{n}\right\}$ is not a Cauchy sequence, then there exist $c \in \operatorname{int}(P)$ and two subsequences $\left\{x_{m(k)}\right\}$ and $\left\{x_{n(k)}\right\}$ of $\left\{x_{n}\right\}$ such that $m(k)$ is the smallest index for which $m(k)>$ $n(k)>k$,

$$
\phi(c)-d\left(x_{m(k)}, x_{n(k)}\right) \notin \operatorname{int}(P)
$$

and

$$
\phi(c)-d\left(x_{m(k)-1}, x_{n(k)}\right) \in \operatorname{int}(P)
$$

Moreover if $\lim _{n \rightarrow \infty} d\left(x_{n}, x_{n+1}\right)=0$, then we have

(1) $\lim _{n \rightarrow \infty} d\left(x_{m(k)}, x_{n(k)}\right)=\phi(c)$;

(2) $\lim _{n \rightarrow \infty} d\left(x_{m(k)+1}, x_{n(k)+1}\right)=\phi(c)$.

Let $(X, d)$ be a cone metric space.

A map $T: X \longrightarrow X$ is called continuous at $x \in X$ if for any $V \in \tau$ containing $T x$, there exists $U \in \tau$ containing $x$ such that $T U \subset V$, where $\tau$ is the topology on $X$ induced by the cone metric $d$. That is,

$\tau=\{U \subset X: \forall x \in U, \exists B \in \beta, x \in B \subset U\}$,

$\beta=\{B(x, c): x \in X, \forall c \in \operatorname{int}(P)\}$,

$B(x, c)=\{y \in X: d(x, y) \ll c\}$. 
If $T$ is continuous at each point $x \in X$, then it is called continuous.

Note that $T$ is continuous if and only if it is sequentially continuous, i.e., $\lim _{n \rightarrow \infty} T x_{n}=T x$ for any sequence $\left\{x_{n}\right\} \subset X$ with $\lim _{n \rightarrow \infty} x_{n}=x$ (see [12]).

A point $x \in X$ is called cluster point of $A \subset X$ if for every $c \in \mathscr{A}$ with $0 \ll c$,

$$
(B(x, c)-\{x\}) \cap A \neq \emptyset .
$$

Let $\Psi$ be the family of all continuous function $\psi: P \longrightarrow P$ such that

$(\psi 1) \psi$ is strictly increasing, i.e., $x<y \Longleftrightarrow \psi(x)<$ $\psi(y)$;

$(\psi 2) \psi^{-1}(\{0\})=0$.

Note that if $\psi(x) \leq \psi(y)$, then $x \leq y$.

Alizadeh et al. [13] introduced the concept of cyclic $(\alpha, \beta)$ admissible mappings in $\mathbb{R}_{+}$.

We extend this concept to cones as follows.

Let $\alpha, \beta: X \longrightarrow P$ be functions, where $X$ is a set. We say that $T: X \longrightarrow X$ is a cyclic $(\alpha, \beta)$-admissible mapping if

(1) $\alpha(x)-e \in P$, where $x \in X \Longrightarrow \beta(T x)-e \in P$;

(2) $\beta(x)-e \in P$, where $x \in X \Longrightarrow \alpha(T x)-e \in P$.

Example 7. Let $K$ be a compact Hausdorff topological space, and let $\mathscr{A}$ be the family of all continuous function from $K$ into $\mathbb{R}$.

Define the multiplication $x y$ of $x$ and $y$ as follows:

$$
x y=x(t) y(t) \quad \forall t \in K \text {. }
$$

Then $\mathscr{A}$ is a Banach algebra with unit $e(t)=1 \forall t \in K$.

Let $P=\{x \in \mathscr{A}: x(t) \geq 0 \forall t \in K\}$.

Then $P$ is a normal cone with normal constant $M=1$. by

Let $X=\mathscr{A}$, and let $\alpha, \beta: X \longrightarrow P$ be functions defined

$$
\alpha(x)=\beta(x)=x \vee e .
$$

Define $T: X \longrightarrow X$ as follows:

$$
T x=x \vee 2 .
$$

Then we have

$$
\begin{gathered}
\alpha(x)-e \in P, \quad x \in P \Longrightarrow \\
\beta(T x)-e=x \vee 2-e \in P
\end{gathered}
$$

and

$$
\begin{gathered}
\beta(x)-e \in P, \quad x \in P \Longrightarrow \\
\alpha(T x)-e=x \vee 2-e \in P .
\end{gathered}
$$

Hence $T$ is cyclic $(\alpha, \beta)$-admissible.

\section{Fixed Points}

Let $\mathscr{C}$ be a family of all continuous functions $f: P \times P \longrightarrow P$ such that

(1) $f(s, t) \leq s \forall s, t \in P$;

(2) $f(s, t)=s \Longrightarrow$ either $s=0$ or $t=0 \forall s, t \in P$.

Then we say that $f \in \mathscr{C}$ is $C$-class function.

Note that $f(0,0)=0$.

Example 8. Let $\mathscr{A}$ be a Banach algebra, and let $P \subset \mathscr{A}$ be a cone.

(1) Let $f_{b}(s, t)=k s$, where $k \in P-\{0\}$ with $\rho(k)<1$.

Since $k \in P-\{0\}$ and $\rho(k)<1$, from Remark $4 e-k \in$ $G(P)$. Hence $s-k s=s(e-k) \in P \forall s \in P$, and hence $0 \leq s-k s$. So $k s \leq s \forall s \in P$. Thus $f_{b}(s, t)=k s \leq$ $s \forall s, t \in P$.

If $f_{b}(s, t)=s$, then $k s=s$, and so $s(e-k)=0 \in P$. Since $e-k \in G(\mathscr{A}), e-k \neq 0$. Hence $s=0$. Thus $f_{b} \in \mathscr{C}$.

(2) Let $f_{b w}(s, t)=\varphi(s)$, where $\varphi: P \longrightarrow P$ is a continuous function such that $\varphi^{-1}(\{0\})=0$ and $\varphi(s)<s \forall t \in$ $P-\{0\}$.

Then $f_{b w}(s, t)=\varphi(s) \leq s \forall s, t \in P$.

Let $f_{b w}(s, t)=s$.

Then $s=\varphi(s)$. If $s \neq 0$, then $s=\varphi(s)<s$, which is a contradiction.

Hence $s=0$. Thus $f_{b w} \in \mathscr{C}$.

(3) Let $f_{w}(s, t)=s-\phi(s)$, where $\phi \in \Phi$.

Then $f_{w}(s, t) \leq s \forall s, t \in P$.

If $f_{w}(s, t)=s$, then $\phi(s)=0$, and so $s=0$. Hence $f_{w} \in \mathscr{C}$.

(4) Let $f_{r}(s, t)=s \beta(s) \forall s, t \in P$,

where $\beta: P \times P \longrightarrow B=\{x \in P-\{0\}: \rho(x)<1\}$ is continuous.

Since $\beta(s) \in P-\{0\}$ and $\rho(\beta(s))<1, e-\beta(s) \in G(P)$. Hence $s-\beta(s) s=s(e-\beta(s)) \in P \forall s \in P$, and hence $\beta(s) s \leq s \forall s \in P$. Thus $f_{r}(s, t)=s \beta(s) \leq s \forall s, t \in P$.

If $f_{r}(s, t)=s$, then $s(e-\beta(s))=s-s \beta(s)=0 \in P$. Since $e-\beta(s) \in G(P), e-\beta(s) \neq 0$. Hence $s=0$.

(5) Let $f_{h}(s, t)=\operatorname{sh}(s, t) \forall s, t \in P$,

where $h: P \times P \longrightarrow B=\{x \in P-\{0\}: \rho(x)<1\}$ is continuous.

Since $h(s, t) \in P-\{0\}$ and $\rho(h(s, t))<1, e-h(s, t) \in$ $G(P)$. Hence $s-h(s, t) s=s(e-h(s, t)) \in P$, and hence $s h(s, t) \leq s$. Thus $f_{h}(s, t)=\operatorname{sh}(s, t) \leq s \forall s, t \in P$.

If $f_{h}(s, t)=s$, then $s(e-h(s, t))=s-s h(s, t)=0 \in P$.

We have $e-h(s, t) \neq 0$, because $e-h(s, t) \in G(P)$.

Hence $s=0$, Thus $f_{h} \in \mathscr{C}$. 
From now on, let $X$ be a cone metric space with cone metric $d$ and regular cone $P$ such that for $x, y \in X$ with $x \neq y$

$$
d(x, y) \in \operatorname{int}(P) .
$$

Theorem 9. Let $X$ be complete, and let $T: X \longrightarrow X$ be a mapping such that

$$
\psi(d(T x, T y)) \leq f(\psi(d(x, y)), \phi(d(x, y)))
$$

for all $x, y \in X$ with $\alpha(x) \beta(y)-e \in P$, where $f \in \mathscr{C}, \psi \in \Psi$, and $\phi \in \Phi$.

Also, suppose that the following are satisfied:

(1) $T$ is cyclic $(\alpha, \beta)$-admissible;

(2) There exists $x_{0} \in X$ such that $\alpha\left(x_{0}\right)-e \in P$ and $\beta\left(x_{0}\right)-$ $e \in P$;

(3) Either $T$ is continuous or if $\left\{x_{n}\right\} \subset X$ is a sequence with $\beta\left(x_{n}\right)-e \in P \forall n=1,2,3, \cdots$ and $x$ is a cluster point of $\left\{x_{n}\right\}$, then

$$
\beta(x)-e \in P .
$$

Then $\mathrm{T}$ has a fixed point.

Moreover, if

$$
\begin{array}{r}
\alpha(x)-e \in P \\
\text { and } \beta(y)-e \in P
\end{array}
$$

for all fixed points $x, y$ of $T$, then $T$ has a unique fixed point.

Proof. Define a sequence $\left\{x_{n}\right\}$ in $X$ by $x_{n}=T x_{n-1}=T^{n} x_{0}$ for all $n=1,2,3, \cdots$.

If there exists an integer $N$ such that $x_{N}=x_{N+1}$, then $x_{N}=T x_{N}$; i.e., $x_{N}$ is a fixed point of $T$.

Hence we assume that $x_{n} \neq x_{n+1} \forall n=1,2,3, \cdots$.

Since $\alpha\left(x_{0}\right)-e \in P$, from (1) we have $\beta\left(x_{1}\right)-e=\beta\left(T x_{0}\right)-$ $e \in P$. Again from (1) we obtain $\alpha\left(x_{2}\right)-e=\alpha\left(T x_{1}\right)-e \in P$.

Inductively, we have

$$
\begin{array}{r}
\alpha\left(x_{2 n}\right)-e \in P \\
\text { and } \beta\left(x_{2 n+1}\right)-e \in P
\end{array}
$$

$$
\forall n=0,1,2, \cdots .
$$

Since $T$ is cyclic $(\alpha, \beta)$-admissible and $\beta\left(x_{0}\right)-e \in P$, $\alpha\left(x_{1}\right)-e=\alpha\left(T x_{0}\right)-e \in P$. Similarly, we obtain

$$
\begin{aligned}
& \quad \beta\left(x_{2 n}\right)-e \in P \\
& \text { and } \alpha\left(x_{2 n+1}\right)-e \in P \\
& \forall n=0,1,2, \cdots .
\end{aligned}
$$

Hence

$$
\begin{aligned}
& \quad \alpha\left(x_{n}\right)-e \in P \\
& \text { and } \beta\left(x_{n}\right)-e \in P \\
& \qquad \forall n=0,1,2, \cdots .
\end{aligned}
$$

Since $\alpha\left(x_{n+1}\right)-e \in P$ and $\beta\left(x_{n}\right)-e \in P$,

$$
\begin{gathered}
\alpha\left(x_{n+1}\right) \beta\left(x_{n}\right)-\alpha\left(x_{n+1}\right)-\beta\left(x_{n}\right)+e \\
=\left(\alpha\left(x_{n+1}\right)-e\right)\left(\beta\left(x_{n}\right)-e\right) \in P .
\end{gathered}
$$

Also,

$$
\begin{aligned}
& \alpha\left(x_{n+1}\right)+\beta\left(x_{n}\right)-2 e=\left(\alpha\left(x_{n+1}\right)-e\right)+\left(\beta\left(x_{n}\right)-e\right) \\
& \quad \in P .
\end{aligned}
$$

Hence,

$$
\alpha\left(x_{n+1}\right) \beta\left(x_{n}\right)-e \in P \quad \forall n=1,2,3, \cdots
$$

And so it follows from (14) that

$$
\begin{aligned}
& \psi\left(d\left(x_{n}, x_{n+1}\right)\right)=\psi\left(d\left(T x_{n-1}, T x_{n}\right)\right) \\
& \quad \leq f\left(\psi\left(d\left(x_{n-1}, x_{n}\right)\right), \phi\left(d\left(x_{n-1}, x_{n}\right)\right)\right) \\
& \quad \leq \psi\left(d\left(x_{n-1}, x_{n}\right)\right) \quad \forall n=1,2,3, \cdots .
\end{aligned}
$$

Since $\psi$ is strictly increasing,

$$
d\left(x_{n}, x_{n+1}\right) \leq d\left(x_{n-1}, x_{n}\right) \quad \forall n=1,2,3, \cdots .
$$

Hence $\left\{d\left(x_{n-1}, x_{n}\right)\right\}$ is a decreasing sequence.

Since $P$ is regular, there exists $r \in P$ such that

$$
\lim _{n \rightarrow \infty} d\left(x_{n-1}, x_{n}\right)=r .
$$

By Lemma 3.1 of [14], we have

$$
r \in \operatorname{int}(P) \cup\{0\} .
$$

Assume that $r \in \operatorname{int}(P)$.

By taking limit $n \longrightarrow \infty$ in (23) and using continuity of $f, \psi$, and $\phi$, we obtain

$$
\psi(r) \leq f(\psi(r), \phi(r)) \leq \psi(r)
$$

which implies

$$
\psi(r)-f(\psi(r), \phi(r)) \in P \cap-P .
$$

So

$$
f(\psi(r), \phi(r))=\psi(r),
$$

and so either $\psi(r)=0$ or $\phi(r)=0$. Hence $r=0$, which is a contradiction.

Hence

$$
r \notin \operatorname{int}(P) .
$$

Thus we have

$$
\lim _{n \rightarrow \infty} d\left(x_{n}, x_{n-1}\right)=0 .
$$

We now show that $\left\{x_{n}\right\}$ is a Cauchy sequence.

On the contrary, assume that $\left\{x_{n}\right\}$ is not a Cauchy sequence. 
By Lemma 6, there exists $c \in \operatorname{int}(P)$ and two subsequences $\left\{x_{m(k)}\right\}$ and $\left\{x_{n(k)}\right\}$ of $\left\{x_{n}\right\}$ such that $m(k)$ is the smallest index for which, for all $k \in \mathbb{N}, m(k)>n(k)>k,(5)$ and (6) hold.

It follows from (19) that $\alpha\left(x_{m(k)}\right) \beta\left(x_{n(k)}\right)-e \in P$, and so from (14) with $x=x_{m(k)}$ and $y=x_{n(k)}$ we have

$$
\begin{aligned}
& \psi\left(d\left(x_{m(k)+1}, x_{n(k)+1}\right)\right)=\psi\left(d\left(T x_{m(k)}, T x_{n(k)}\right)\right) \\
& \quad \leq f\left(\psi\left(d\left(x_{m(k)}, x_{n(k)}\right)\right), \phi\left(d\left(x_{m(k)}, x_{n(k)}\right)\right)\right) .
\end{aligned}
$$

By Letting $k \longrightarrow \infty$ and by using Lemma 6 (1) and (2), and using continuity of $\psi, \phi$, and $f$, we have

$$
\psi(\phi(c)) \leq f(\psi(\phi(c)), \phi(\phi(c))) \leq \psi(\phi(c))
$$

which implies

$$
\psi(\phi(c))-f(\psi(\phi(c)), \phi(\phi(c))) \in P \cap-P .
$$

Hence

$$
f(\psi(\phi(c)), \phi(\phi(c)))=\psi(\phi(c))
$$

which implies either $\psi(\phi(c))=0$ or $\phi(\phi(c))=0$. Thus $\phi(c)=$ 0 , and so $c=0$, which is a contradiction.

Therefore, $\left\{x_{n}\right\}$ is a Cauchy sequence.

It follows from the completeness of $X$ that there exists

$$
x_{*}=\lim _{n \longrightarrow \infty} x_{n} \in X .
$$
$T x_{*}$.

If $T$ is continuous, then $\lim _{n \rightarrow \infty} x_{n}=T x_{*}$, and so $x_{*}=$

Assume that (15) holds.

Then we have

$$
\beta\left(x_{*}\right)-e \in P .
$$

Since $T$ is $(\alpha, \beta)$-admissible, $\alpha\left(x_{n}\right)-e \in P \forall n=1,2,3$,

Using (19) we have

$$
\alpha\left(x_{n}\right) \beta\left(x_{*}\right)-e \in P \quad \forall n=1,2,3, \cdots .
$$

From (14) we have

$$
\begin{aligned}
& \psi\left(d\left(x_{n+1}, T x_{*}\right)\right)=\psi\left(d\left(T x_{n}, T x_{*}\right)\right) \\
& \quad \leq f\left(\psi\left(d\left(x_{n}, x_{*}\right)\right), \phi\left(d\left(x_{n}, x_{*}\right)\right)\right) .
\end{aligned}
$$

Letting $n \longrightarrow \infty$ in the inequality (39) and using continuity of $f, \psi$, and $\phi$, we have

$$
\begin{aligned}
& \psi\left(d\left(x_{*}, T x_{*}\right)\right) \leq f(\psi(0), \phi(0))=0, \\
& \text { and so } \psi\left(d\left(x_{*}, T x_{*}\right)\right) \in-P
\end{aligned}
$$

which implies

$$
\psi\left(d\left(x_{*}, T x_{*}\right)\right) \in P \cap-P .
$$

Thus

$$
\psi\left(d\left(x_{*}, T x_{*}\right)\right)=0 .
$$

Hence

$$
\begin{aligned}
d\left(x_{*}, T x_{*}\right) & =0, \\
\text { and hence } x_{*} & =T x_{*} .
\end{aligned}
$$

For the uniqueness of fixed point, assume that (16) holds and that $y_{*}$ is another fixed point of $T$.

Then from (16) we have $\alpha\left(x_{*}\right)-e \in P$ and $\beta\left(y_{*}\right)-e \in P$. Hence $\alpha\left(x_{*}\right) \beta\left(y_{*}\right)-e \in P$.

It follows from (14) that

$$
\begin{aligned}
\psi\left(d\left(x_{*}, y_{*}\right)\right) & =\psi\left(d\left(T x_{*}, T y_{*}\right)\right) \\
& \leq f\left(\psi\left(d\left(x_{*}, y_{*}\right)\right), \phi\left(d\left(x_{*}, y_{*}\right)\right)\right) \\
& \leq \psi\left(d\left(x_{*}, y_{*}\right)\right)
\end{aligned}
$$

which implies

$$
\begin{aligned}
& \psi\left(d\left(x_{*}, y_{*}\right)\right)-f\left(\psi\left(d\left(x_{*}, y_{*}\right)\right), \phi\left(d\left(x_{*}, y_{*}\right)\right)\right) \\
& \quad \in P \cap-P .
\end{aligned}
$$

Thus

$$
f\left(\psi\left(d\left(x_{*}, y_{*}\right)\right), \phi\left(d\left(x_{*}, y_{*}\right)\right)\right)=\psi\left(d\left(x_{*}, y_{*}\right)\right)
$$

which implies

$$
\begin{array}{r}
\text { either } \psi\left(d\left(x_{*}, y_{*}\right)\right)=0 \\
\text { or } \phi\left(d\left(x_{*}, y_{*}\right)\right)=0 .
\end{array}
$$

So $d\left(x_{*}, y_{*}\right)=0$, and hence $x_{*}=y_{*}$.

Example 10. Let $\mathscr{A}=\mathbb{R}^{2}, P=\left\{\left(x_{1}, x_{2}\right) \in \mathscr{A}: x_{1}, x_{2} \geq 0\right\}$, and let $\|x\|=\left|x_{1}\right|+\left|x_{2}\right| \forall x=\left(x_{1}, x_{2}\right) \in \mathscr{A}$.

Define the multiplication $x y$ of $x=\left(x_{1}, x_{2}\right)$ and $y=$ $\left(y_{1}, y_{2}\right)$ as follows:

$$
x y=\left(x_{1}, x_{2}\right)\left(y_{1}, y_{2}\right)=\left(x_{1} y_{1}, x_{1} y_{2}+x_{2} y_{1}\right) .
$$

Then $\mathscr{A}$ is a Banach algebra with unit $e=(1,0), P$ is regular cone, and $\operatorname{int}(P)=\left\{\left(x_{1}, x_{2}\right) \in \mathscr{A}: x_{1}, x_{2}>0\right\}$.

Let $X=\mathbb{R}^{2}$, and let $d: X \times X \longrightarrow \mathscr{A}$ be a mapping defined by

$$
d(x, y)=\max \left\{\left|x_{1}-y_{1}\right|,\left|x_{2}-y_{2}\right|\right\} .
$$

Then $(X, d)$ is a complete cone metric space, and $d(x, y) \in$ $\operatorname{int}(P) \forall x, y \in X$ with $x \neq y$.

Define a mapping $T: X \longrightarrow X$ by

$T x$

$$
= \begin{cases}\frac{1}{4}\left(x_{1}, x_{2}\right), & x=\left(x_{1}, x_{2}\right) \in P \text { and }\|x\|<1 ; \\ \left(\log \left|x_{1} x_{2}\right|, e^{x_{1} x_{2}}\right), & \text { otherwise. }\end{cases}
$$

Let $\varphi(t)=(1 / 2) t, \psi(t)=t$, and $\phi(t)=(1 / 3) t \forall t=\left(t_{1}\right.$, $\left.t_{2}\right) \in P$ 
We define two functions $\alpha, \beta: X \longrightarrow P$ by

$\alpha(x)=\beta(x)= \begin{cases}e & x=\left(x_{1}, x_{2}\right) \in P \text { and }\|x\|<1 \\ 0 & \text { otherwise. }\end{cases}$

Obviously, $T$ is $(\alpha, \beta)$-admissible.

Let $f(s, t)=f_{b w}(s, t) \forall s, t \in P$.

Then we have

$$
\begin{aligned}
& f_{b w}(\psi(d(x, y)), \phi(d(x, y)))-\psi(d(T x, T y)) \\
&= f_{b w}(d(x, y), \phi(d(x, y)))-d(T x, T y) \\
&= \varphi(d(x, y))-d(T x, T y) \\
&= \frac{1}{2} \max \left\{\left|x_{1}-y_{1}\right|,\left|x_{2}-y_{2}\right|\right\} \\
&-d\left(\frac{1}{4}\left(x_{1}, x_{2}\right), \frac{1}{4}\left(y_{1}, y_{2}\right)\right) \\
&= \frac{1}{2} \max \left\{\left|x_{1}-y_{1}\right|,\left|x_{2}-y_{2}\right|\right\} \\
&-\frac{1}{4} \max \left\{\left|x_{1}-y_{1}\right|,\left|x_{2}-y_{2}\right|\right\} \\
&= \frac{1}{4} \max \left\{\left|x_{1}-y_{1}\right|,\left|x_{2}-y_{2}\right|\right\} \in P \\
& \quad \forall x, y \in X \text { with } \alpha(x) \beta(y)-e \in P .
\end{aligned}
$$

Hence $\psi(d(T x, T y)) \leq f_{b w}(\psi(d(x, y)), \phi(d(x, y))) \forall x$, $y \in X$ with $\alpha(x) \beta(y)-e \in P$.

Condition (2) of Theorem 9 is satisfied with $x_{0}=(0,0)$.

We now show condition (15) of Theorem 9.

Let $x_{n}=(1 /(n+4), 2 /(n+4)) \forall n=1,2,3, \cdots$.

Then $x_{n} \in P$ and $\left\|x_{n}\right\|<1 \forall n=1,2,3, \cdots$. Hence

$$
\beta\left(x_{n}\right)-e=(0,0) \in P \quad \forall n=1,2,3, \cdots .
$$

Then $\lim _{n \rightarrow \infty} x_{n}=(0,0)$, and so $(0,0)$ is a cluster point of $\left\{x_{n}\right\}$.

Then we obtain

$$
\beta((0,0))-e=(0,0) \in P .
$$

Hence condition (15) of Theorem 9 is satisfied.

Thus, all hypotheses of Theorem 9 are satisfied and $T$ has a fixed point $x_{*}=(0,0)$.

Corollary 11. Let $X$ be complete, and let $T: X \longrightarrow X$ be a mapping such that

$$
\begin{aligned}
& \psi(\alpha(x) \beta(y) d(T x, T y)) \\
& \quad \leq f(\psi(d(x, y)), \phi(d(x, y)))
\end{aligned}
$$

for all $x, y \in X$, where $f \in \mathscr{C}, \psi \in \Psi$, and $\phi \in \Phi$. hold.

Also, suppose that conditions (1), (2), and (3) of Theorem 9

Then $T$ has a fixed point.

Moreover, if (16) holds, then T has a unique fixed point.

\section{Discussion}

We give an application of Theorem 9 to prove existence of fixed points for cyclic mappings.

Theorem 12. Let $X$ be complete, and let $A$ and $B$ be two closed subsets of $X$ such that $A \cap B \neq \emptyset$, and let $T: A \cup B \longrightarrow A \cup B$ be a mapping such that

$$
T A \subset B
$$

and $T B \subset A$.

\section{Assume that}

$$
\psi(d(T x, T y)) \leq f(\psi(d(x, y)), \phi(d(x, y)))
$$

for all $x \in A$ and $y \in B$, where $f \in \mathscr{C}, \psi \in \Psi$, and $\phi \in \Phi$.

Then $T$ has a unique fixed point in $A \cap B$.

Proof. Define $\alpha, \beta: X \longrightarrow P$ by

$$
\begin{aligned}
& \alpha(x)= \begin{cases}e, & x \in A \\
0, & \text { otherwise, }\end{cases} \\
& \beta(x)= \begin{cases}e, & x \in B \\
0, & \text { otherwise. }\end{cases}
\end{aligned}
$$

Then we have that $\forall x, y \in A \cup B$

$$
\begin{aligned}
& \alpha(x) \beta(y)-e \in P \Longleftrightarrow \\
& e \leq \alpha(x) \beta(y) \Longleftrightarrow
\end{aligned}
$$

Hence (57) implies (13).

It follows from (56), (58), and (59) that

$$
\begin{gathered}
\alpha(x)-e \in P \Longrightarrow \\
e \leq \alpha(x) \Longrightarrow \\
x \in A \Longrightarrow \\
T x \in B \Longrightarrow \\
e \leq \beta(T x) \Longrightarrow \\
\beta(T x)-e \in P
\end{gathered}
$$

and

$$
\begin{gathered}
\beta(x)-e \in P \Longrightarrow \\
e \leq \beta(x) \Longrightarrow \\
x \in B \Longrightarrow \\
T x \in A \Longrightarrow \\
e \leq \alpha(T x) \Longrightarrow \\
\alpha(T x)-e \in P .
\end{gathered}
$$


Thus $T$ is cyclic $(\alpha, \beta)$-admissible.

Because $A \cap B \neq \emptyset$, there exists an $x_{0}$ in $A \cap B$. By definition of functions $\alpha$ and $\beta, \alpha\left(x_{0}\right)-e \in P$ and $\beta\left(x_{0}\right)-e \in P$. Hence condition (2) of Theorem 9 is satisfied.

Let $\left\{x_{n}\right\} \subset X$ be a sequence such that

$$
\begin{aligned}
& \lim _{n \rightarrow \infty} d\left(x_{n}, x\right)=0 \\
& \text { and } \beta\left(x_{n}\right)-e \in P
\end{aligned}
$$

$$
\forall n=1,2,3, \cdots .
$$

Then $x_{n} \in B, \forall n=1,2,3, \cdots$. Since $B$ is closed, $x \in B$ and so $\beta(x)-e \in P$. Hence condition (3) of Theorem 9 holds.

Thus all conditions of Theorem 9 are satisfied. It follows from Theorem 9 that $T$ has a fixed point in $A \cup B$; say $x_{*} \in$ $A \cup B$.

If $x_{*} \in A$, then from (56) $x_{*}=T x_{*} \in B$. Thus $x_{*} \in A \cap B$. Similarly, we have $x_{*} \in A \cap B$, when $x_{*} \in B$.

Let $x, y$ be any two fixed point of $T$.

Then $x, y \in A \cap B$, and so $\alpha(x) \beta(y)-e \in P$. It follows from Theorem 9 that $T$ has a unique fixed point in $A \cap B$.

Corollary 13. Let $X$ be complete, and let $A$ and $B$ be two closed subsets of $X$ such that $A \cap B \neq \emptyset$, and let $T: A \cup B \longrightarrow A \cup B$ be a mapping such that

$$
T A \subset B
$$

and $T B \subset A$.

Assume that

$$
d(T x, T y) \leq f(d(x, y), \phi(d(x, y)))
$$

for all $x \in A$ and $y \in B$, where $f \in \mathscr{C}$ and $\phi \in \Phi$.

Then $T$ has a unique fixed point in $A \cap B$.

Corollary 14. Let $X$ be complete, and let $A$ and $B$ be two closed subsets of $X$ such that $A \cap B \neq \emptyset$, and let $T: A \cup B \longrightarrow A \cup B$ be a mapping such that

$$
T A \subset B
$$

and $T B \subset A$

Assume that

$$
d(T x, T y) \leq k d(x, y)
$$

for all $x \in A$ and $y \in B$, where $k \in P-\{0\}$ and $\rho(k)<1$.

Then $T$ has a unique fixed point in $A \cap B$.

Proof. From (67) we have

$$
\begin{aligned}
& d(T x, T y) \leq k d(x, y) \Longleftrightarrow \\
& d(T x, T y) \leq f_{b}(d(x, y), \phi(d(x, y))
\end{aligned}
$$

for all $x \in A$ and $y \in B$, where $k \in P-\{0\}, \rho(k)<1$, and $\phi \in \Phi$

By Corollary 13 with $f(s, t)=f_{b}(s, t) \forall s, t \in P, T$ has a unique fixed point in $A \cap B$.
Remark 15. If $\mathscr{A}=\mathbb{R}$ and $P=[0, \infty)$, then Corollary 14 reduces to Theorem 1.1 of [15].

Remark 16. Corollary 14 is a generalization of Theorem 2.1 of [7] (resp., Theorem 3.1 of [16]) to cyclic mappings under assumption of regularity.

\section{Applications}

By applying $C$-class functions of Example 8 to Theorem 9, we derive some existing fixed point results in the literature and have generalizations of well-known fixed point theorems in metric spaces to cone metric spaces.

In particular, by taking $f(s, t)=f_{w}(s, t)$, we have the following result.

Theorem 17. Let $X$ be complete, and let $T: X \longrightarrow X$ be a $\operatorname{cyclic}(\alpha, \beta)$-admissible mapping such that

$$
\psi(d(T x, T y)) \leq \psi(d(x, y))-\phi(d(x, y))
$$

for all $x, y \in X$ with $\alpha(x) \beta(y)-e \in P$, where $\psi \in \Psi$ and $\phi \in \Phi$ such that

$$
\phi(\psi(t)) \leq \phi(t) \quad \forall t>0 .
$$

Suppose that $\alpha\left(x_{0}\right)-e \in P$ and $\beta\left(x_{0}\right)-e \in P$, where $x_{0} \in X$.

Assume that either

(1) $T$ is continuous or

(2) if $\left\{x_{n}\right\} \subset X$ is a sequence such that $\lim _{n \rightarrow \infty} d\left(x_{n}, x\right)=$ 0 and $\beta\left(x_{n}\right)-e \in P \forall n=1,2,3, \cdots$, then

$$
\beta(x)-e \in P \text {. }
$$

Then $T$ has a fixed point in $X$. Further if $\alpha(x) \beta(y)-e \in P$ for all fixed points $x, y$ of $T$, then $T$ has a unique fixed point.

Proof. Let $f_{w}(s, t)=s-\phi(s) \forall t, s \in P$, where $\phi \in \Phi$.

Then $f_{w}$ is a C-class function.

From (69) and (70) we have that for all $x, y \in X$ with $\alpha(x) \beta(y)-e \in P$

$$
\begin{aligned}
0 & \leq \psi(d(x, y))-\phi(d(x, y))-\psi(d(T x, T y)) \\
& \leq \psi(d(x, y))-\phi(\psi(d(x, y)))-\psi(d(T x, T y)) \\
& =f_{w}(\psi(d(x, y)), \phi(d(x, y)))-\psi(d(T x, T y)) .
\end{aligned}
$$

Hence

$$
\psi(d(T x, T y)) \leq f_{w}(\psi(d(x, y)), \phi(d(x, y)))
$$

for all $x, y \in X$ with $\alpha(x) \beta(y)-e \in P$.

It follows that (13) holds with $f=f_{w}$. All conditions of Theorem 9 hold, and so we have a desired result.

Corollary 18. Let $X$ be complete, and let $T: X \longrightarrow X$ be a cyclic $(\alpha, \beta)$-admissible mapping such that

$$
d(T x, T y) \leq d(x, y)-\phi(d(x, y))
$$

for all $x, y \in X$ with $\alpha(x) \beta(y)-e \in P$, where $\phi \in \Phi$. 
Suppose that $\alpha\left(x_{0}\right)-e \in P$ and $\beta\left(x_{0}\right)-e \in P$, where $x_{0} \in X$.

Assume that either

(1) $T$ is continuous or

(2) if $\left\{x_{n}\right\} \subset X$ is a sequence such that $\lim _{n \rightarrow \infty} d\left(x_{n}, x\right)=$ 0 and $\beta\left(x_{n}\right)-e \in P \forall n=1,2,3, \cdots$, then

$$
\beta(x)-e \in P \text {. }
$$

Then $T$ has a fixed point in X. Further if $\alpha(x) \beta(y)-e \in P$ for all fixed points $x, y$ of $T$, then $T$ has a unique fixed point.

By taking $\alpha(x)=e$ and $\beta(x)=e \forall x \in X$ in Theorem 17, we have the following result.

Corollary 19. Let $X$ be complete, and let $T: X \rightarrow X$ be a mapping such that

$$
\psi(d(T x, T y)) \leq \psi(d(x, y))-\phi(d(x, y))
$$

for all $x, y \in X$, where $\psi \in \Psi$ and $\phi \in \Phi$ with $\phi(\psi(t)) \leq$ $\phi(t) \forall t>0$.

Then $T$ has a unique fixed point in $X$.

Corollary 20. Let $X$ be complete, and let $T: X \rightarrow \mathrm{X}$ be a mapping such that

$$
d(T x, T y) \leq d(x, y)-\phi(d(x, y))
$$

for all $x, y \in X$, where $\phi \in \Phi$.

Then $T$ has a unique fixed point in $X$.

Remark 21. By taking $\mathscr{A}$ a real Banach space in Corollary 19 (resp., Corollary 20), we have Theorem 2.1 of [10] (resp., Theorem 2.1 of [4]).

\section{Data Availability}

The data used to support the findings of this study are available from the corresponding author upon request.

\section{Conflicts of Interest}

The author declares that he has no conflicts of interest.

\section{References}

[1] L. G. Huang and X. Zhang, "Cone metric spaces and fixed point theorems of contractive mappings," Journal of Mathematical Analysis and Applications, vol. 332, no. 2, pp. 1468-1476, 2007.

[2] S.-H. Cho and J.-S. Bae, "Common fixed point theorems for mappings satisfying property (E.A) on cone metric spaces," Mathematical and Computer Modelling, vol. 53, no. 5-6, pp. 945951, 2011.

[3] S. H. Cho and J. S. Bae, "Fixed point theorems for multivalued maps in cone metric spaces," Fixed Point Theory and Applications, vol. 2011, article 87, 2011.

[4] B. S. Choudhury and N. Metiya, "Fixed points of weak contractions in cone metric spaces," Nonlinear Analysis. Theory, Methods \& Applications. An International Multidisciplinary Journal, vol. 72, no. 3-4, pp. 1589-1593, 2010.
[5] S. Rezapour and R. Hamlbarani, "Some notes on the paper "Cone metric spaces and fixed point theorems of contractive mappings"," Journal of Mathematical Analysis and Applications, vol. 345, no. 2, pp. 719-724, 2008.

[6] S. Yang, J. Bae, and S. Cho, "Coincidence and common fixed and periodic point theorems in cone metric spaces," Computers \& Mathematics with Applications, vol. 61, no. 2, pp. 170-177, 2011.

[7] H. Liu and S. Xu, "Cone metric spaces with Banach algebras and fixed point theorems of generalized Lipschitz mappings," Fixed Point Theory and Applications, vol. 2013, article no. 320, 2013.

[8] S. Chandok, K. Tas, and A. H. Ansari, "Some Fixed Point Results for TAC-Type Contractive MappingsContractive Mappings," Journal of Function Spaces, vol. 2016, Article ID 1907676, 6 pages, 2016.

[9] W. Rudin, Functional Analysis and Its Applications, McGrawHill, New York, NY, USA, 1991.

[10] C. T. Aage and J. N. Salunke, "Fixed points of $(\psi, \Phi)$-weak contractions in cone metric spaces," Annals of Functional Analysis, vol. 2, no. 1, pp. 59-71, 2011.

[11] B. S. Choudhury and N. Metiya, "Fixed point and common fixed point results in ordered cone metric spaces," Analele stiintifice ale Universitatii Ovidius Constanta, vol. 20, no. 1, pp. 55-72, 2012.

[12] D. Turkoglu and M. Abuloha, "Cone metric spaces and fixed point theorems in diametrically contractive mappings," Acta Mathematica Sinica, vol. 26, no. 3, pp. 489-496, 2010.

[13] A. A. Sattar, M. Fridoun, and S. Peyman, "Some fixed point results for $(\alpha, \beta)-(\psi, \phi)$-contractive mappings," Filomat, vol. 28, no. 3, pp. 635-647, 2014.

[14] B. S. Choudhury and N. Metiya, "The point of coincidence and common fixed point for pair of mappings in cone metric spaces," Computers and Mathematics with Apllications, vol. 60, pp. 1686-1695, 2010.

[15] W. A. Kirk, P. S. Srinivasan, and P. Veeramani, "Fixed points for mappings satisfying cyclical contractive conditions," Fixed Point Theory and Applications, vol. 4, no. 1, pp. 79-89, 2003.

[16] S. Xu and S. Radenović, "Fixed point theorems of generalized Lipschitz mappings on cone metric spaces over Banach algebras without assumption of normality," Fixed Point Theory and Applications, vol. 2014, no. 1, p. 102, 2014. 


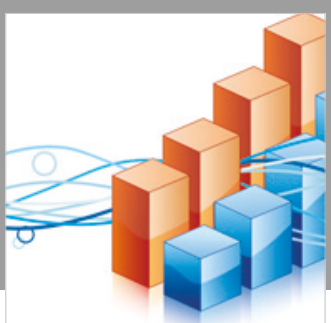

Advances in

Operations Research

\section{-n-m}
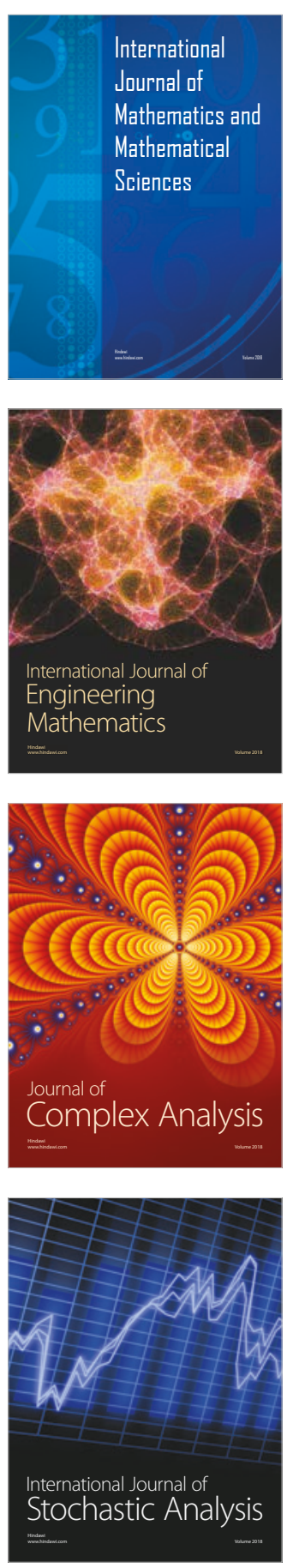
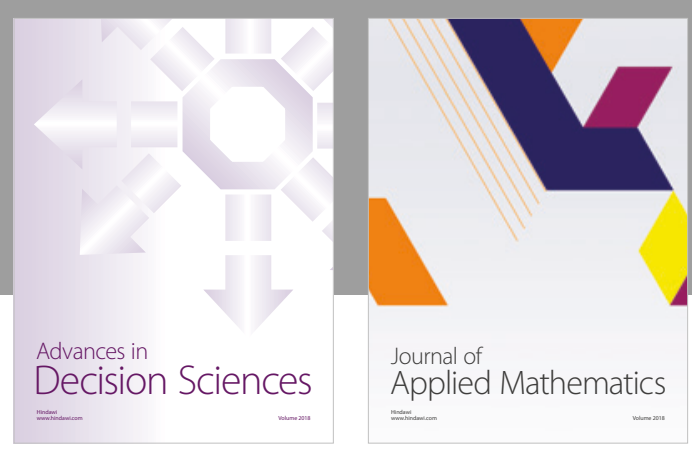

Journal of

Applied Mathematics
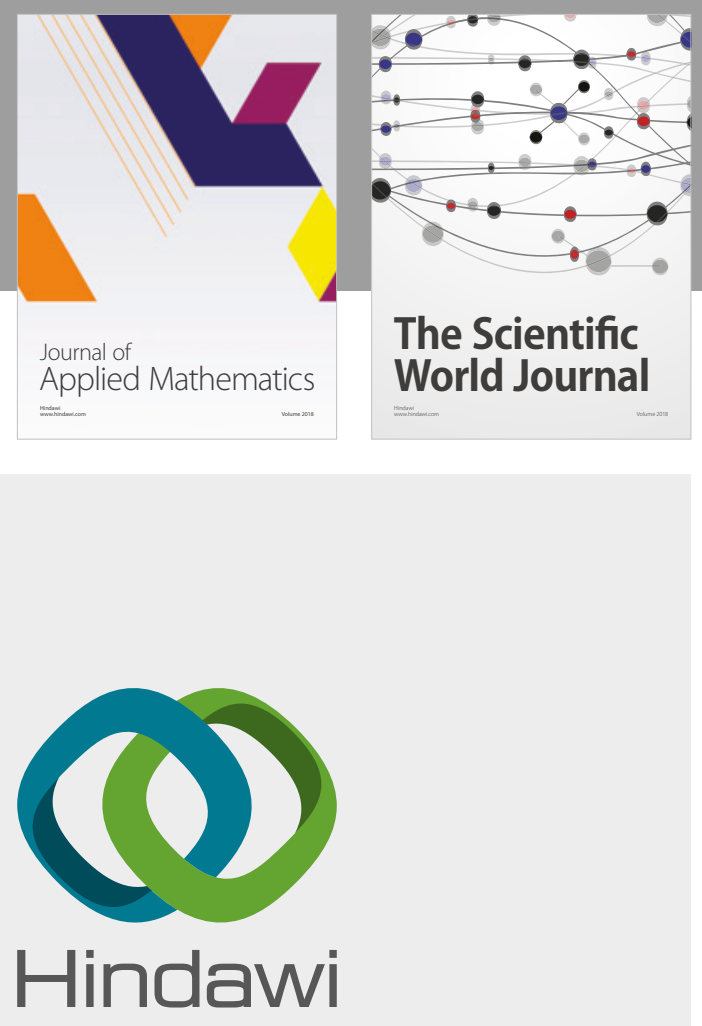

Submit your manuscripts at

www.hindawi.com

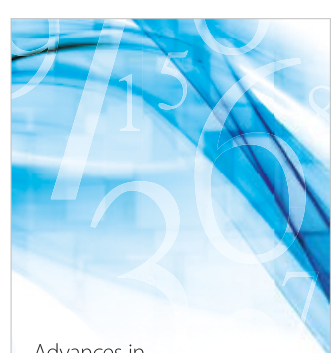

Advances in
Numerical Analysis
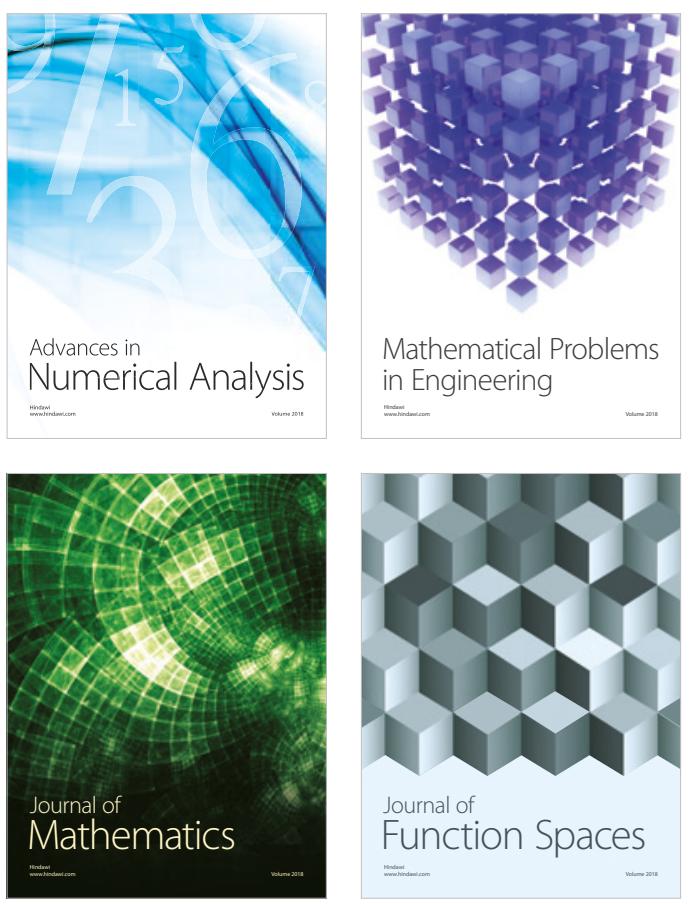

Mathematical Problems in Engineering

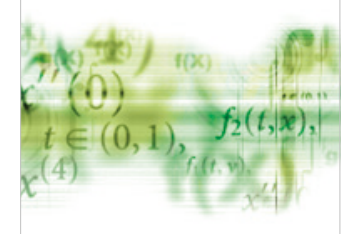

International Journal of

Differential Equations

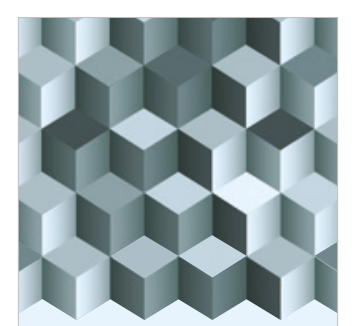

Journal of

Function Spaces

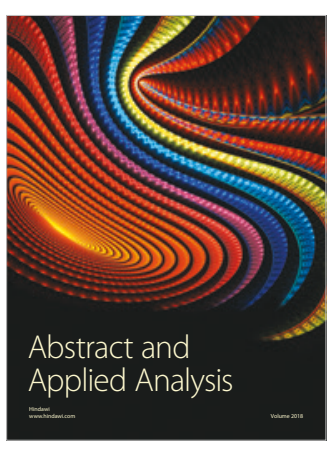

The Scientific

World Journal

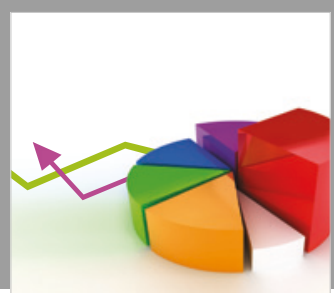

Journal of

Probability and Statistics
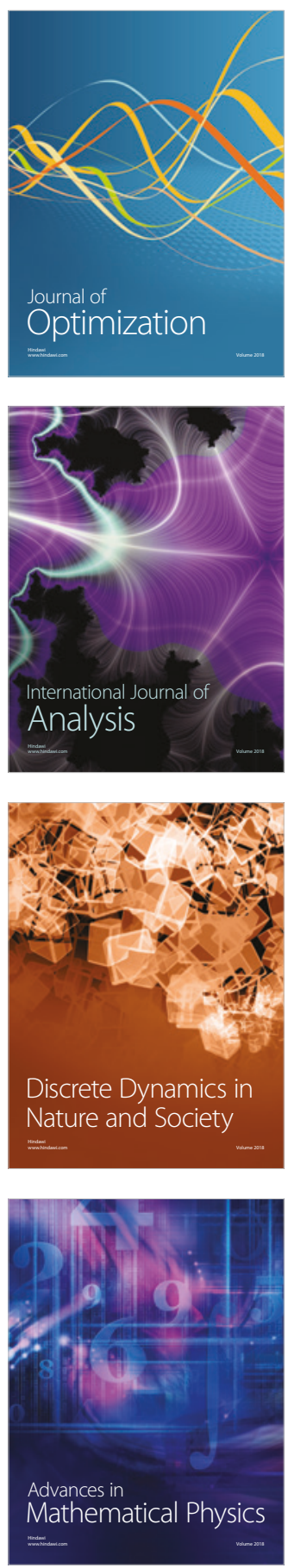